American Journal of Applied Sciences 3 (11): 2103-2107, 2006

ISSN 1546-9239

(C) 2006 Science Publications

\title{
Fast Iterative Frequential Equalization, Application to Mobile Communications
}

\author{
${ }^{1,2}$ M.T. Taba, ${ }^{1}$ S. Femmam and ${ }^{3}$ M. Bedda \\ ${ }^{1}$ Laboratoire LACSC, ECE PARIS, France \\ ${ }^{2}$ Laboratoire LAIG, Guelma University, Algeria \\ ${ }^{3}$ Laboratoire LASA, Annaba University, Algeria
}

\begin{abstract}
The Space Time Adaptive Processing (STAP) is a promising approach in the improvement of the wireless communication systems. Since it makes possible to reduce interferences ISI (Inter Symbol Interference) and CCI (Co-Channel Interference) but its disadvantage is the intensive treatment which reduces the speed of convergence of the algorithm LMS (Least Mean Square) and SMI (Sampling Matrix Inversion). Our approach resides in the use of a fast algorithm which calculate the weighting coefficients of the equalizer in frequential field with faster convergence.
\end{abstract}

Key words: Fast iterative frequential equalization, mobile communications, convergence, equalizer

\section{INTRODUCTION}

Signal processing in wireless communications include modulation / demodulation, coding/decoding, channel equalization and estimation of the transmitted signals and reduction of interferences ISI and CCI. As the mobile communications are developing toward the higher speed digital networks ${ }^{[1]}$, the associated communication channels become severely frequency selective, which makes (ISI) highly pronounced. Additionally, due to frequency reuse (CCI) signals are present against the desired user signal. Therefore, the system capacity and the communication quality are greatly affected by both the ISI and CCI problems ${ }^{[2]}$.

Adaptive arrays, particularly under space-time adaptive processing (STAP) ${ }^{[3,4]}$, provide effective ways to suppress both the ISI and the CCI, subsequently improving the system capacity and the communication quality ${ }^{[3-7]}$. A STAP system Fig. 1 is usually composed of an antenna array and a set of FIR filters after the array elements to perform joint spatial and temporal adaptive processing.

In spite of its performances, a STAP system faces the problems of a high computational burden and a low convergence rate. These problems become particularly serious when operating in the severe fading environments where longer FIR filters are needed ${ }^{[8]}$. For example, when algorithms $\mathrm{LMS}^{[3]}$ are used, the convergence speed becomes extremely slow and thereafter a long training sequence is needed ${ }^{[8]}$.

To solve these problems of convergence we propose an iterative method which operates in the frequencies field.

Signal model: Consider a base station using an antenna array of $\mathrm{N}$ Elements $(\mathrm{N}>=1)$ with $\mathrm{P}(\mathrm{P}>=1)$ users. The signal of the desired user is denoted $\mathrm{s}_{1}(\mathrm{t})$, whereas

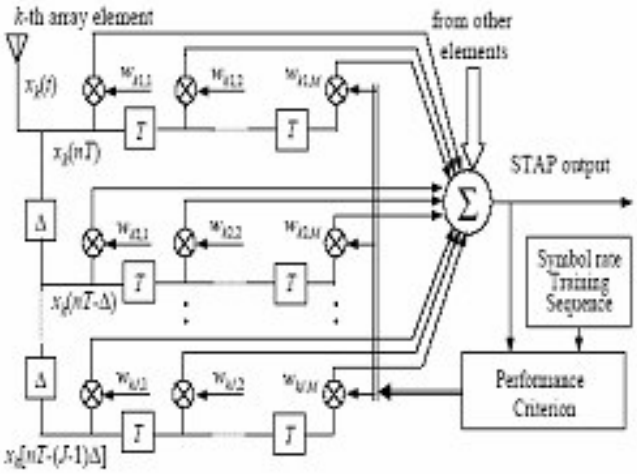

Fig. 1: The scheme of the fractionally spaced STAP

signals from other users are denoted $s_{p}(t), p=2, \ldots, P$. The array output vector $x(t)$ is expressed as:

$$
\begin{aligned}
& \mathrm{x}(\mathrm{t})=\sum_{\mathrm{p}=1}^{\mathrm{p}} \sum_{\mathrm{l}=1}^{\mathrm{Lp}} \mathrm{a}\left(\theta_{1}^{\mathrm{p}}\right) \xi_{1}^{\mathrm{p}} \mathrm{S}_{\mathrm{p}}\left(\mathrm{t}-\tau_{1}^{\mathrm{p}}\right)+\mathrm{n}(\mathrm{t}) \\
& \mathrm{x}(\mathrm{t})=\sum_{\mathrm{p}=1}^{\mathrm{p}} \sum_{\mathrm{m}=-\infty}^{+\infty} \mathrm{S}_{\mathrm{p}}(\mathrm{m}) \mathrm{h}_{\mathrm{p}}(\mathrm{t}-\mathrm{mT})+\mathrm{n}(\mathrm{t})
\end{aligned}
$$

Where

$$
\begin{aligned}
& \mathrm{S}_{\mathrm{p}}(\mathrm{t})=\sum_{\mathrm{m}=-\infty}^{+\infty} \overline{\mathrm{S}_{\mathrm{p}}}(\mathrm{m}) \rho_{\mathrm{p}}(\mathrm{t}-\mathrm{mT}) \\
& \mathrm{h}_{\mathrm{p}}(\mathrm{t})=\sum_{\mathrm{l}=1}^{\mathrm{L}_{\mathrm{p}}} \mathrm{a}\left(\theta_{1}^{\mathrm{p}}\right) \xi_{1}^{\mathrm{p}} \rho\left(\mathrm{t}-\tau_{1}^{\mathrm{p}}\right)
\end{aligned}
$$

where $h_{p}(t)$ represents the space time impulse response of the channel p. and :

$\left\{\theta_{1}^{\mathrm{p}}, \tau_{1}^{\mathrm{p}}, \xi_{1}^{\mathrm{p}}\right\}$ Angle-of-arrival (AOA), time delay and propagation loss corresponding to the $l^{\text {th }}$ path of the $p^{\text {th }}$ user.

$\mathrm{a}(\theta)$ : Array steering vector corresponding to $\theta$.

$\overline{\mathrm{S}_{\mathrm{p}}}(\mathrm{m}): \mathrm{m}^{\text {th }}$ information symbol of the $P^{\text {th }}$ user.

$\rho_{p}(t)$ : Pulse shaping function of the $P^{t h}$ user.

$L_{p}$ : Total number of multipath rays of the $P^{\text {th }}$ user. 
$T$ : Symbol duration.

$n(t)$ : Array noise vector.

At the receiver we sample the signal $\mathrm{x}(\mathrm{t})$ at sampling cycle $\Delta$ and let $\mathrm{J}=\mathrm{T} / \Delta$ be the factor of oversampling $^{[9]}$. Sampling $\mathrm{x}(\mathrm{t})$ at $\mathrm{t}=\mathrm{i} \Delta+\mathrm{nT}$, equation (1) becomes

$\mathrm{x}(\mathrm{i} \Delta+\mathrm{nT})=\sum_{\mathrm{p}=1}^{\mathrm{P}} \sum_{\mathrm{d}=0}^{\mathrm{D}_{\mathrm{p}}} \overline{\mathrm{S}_{\mathrm{p}}}(\mathrm{n}-\mathrm{d}) \mathrm{h}_{\mathrm{p}}(\mathrm{i} \Delta+\mathrm{dT})+\mathrm{n}(\mathrm{i} \Delta+\mathrm{nT})$

With the exploitation of the cyclostationarity of user signals ${ }^{[2]}$, the extended multichannel model of STAP can be established as :

$\mathrm{x}(\mathrm{n})=\sum_{\mathrm{p}=1}^{\mathrm{P}} \sum_{\mathrm{d}=0}^{\mathrm{D}_{\mathrm{p}}} \overline{\mathrm{S}_{\mathrm{p}}}(\mathrm{n}-\mathrm{d}) \mathrm{h}_{\mathrm{p}}(\mathrm{d})+\mathrm{n}(\mathrm{n})$

where: $\alpha(\mathrm{n})=\left[\alpha^{\mathrm{T}}(\mathrm{nT}), \ldots, \alpha^{\mathrm{T}}(\mathrm{nT}+(\mathrm{J}-1) \Delta)\right]^{\mathrm{T}}$

$\alpha$ : represents $x, h$, or $n$.

By considering the consecutive samples for one period of $\mathrm{M}$ symbols, we define the following vectors:

$$
\begin{aligned}
& X(n)=\left[x^{T}(n), x^{T}(n-1), \ldots . x^{T}(n-m+1)\right]^{T} \\
& S_{p}(n)=\left[s_{p}^{T}(n), s_{p}^{T}(n-1), \ldots . s_{p}^{T}\left(n-m-D_{p}+1\right)\right]^{T} \\
& N(n)=\left[n^{T}(n), n^{T}(n-1), \ldots . n^{T}(n-m+1)\right]^{T} \\
& h_{p}=\left[h_{p}^{T}(0), h_{p}^{T}(1), \ldots . . h_{p}^{T}\left(D_{p}\right)\right]^{T}
\end{aligned}
$$

Thus, $X(n)$ can be defined like :

$$
\begin{aligned}
& X(n)=\sum_{p=1}^{P} h_{p} S_{p}(n)+N(n)=H S(n)+N(n) \\
& H=\left[H_{1}, \ldots, H_{p}\right] \text { and } S(n)=\left[S_{1}^{T}(n), \ldots, S_{P}^{T}\right]
\end{aligned}
$$

Where the columns of $\mathrm{H}$ express the space-time response associated with users delayed sequences. The limit of the number of the extended channels by over sampling is discussed in $^{[6]}$.

The MMSE criterion: The output of STAP system ${ }^{[8]}$, as shown in Fig. 1 is defined:

$y(n)=W^{T} X(n)$

where $W$ is the weighting vector of FIR filters. 1t is known that in the absence of the noise, one of the necessary conditions for a perfect adaptive processing $^{[1]}$, i.e, the perfect equalization of the desired signals of the user and the perfect removal of CCI signals, is that the matrix $\mathrm{H}$ is of full row (non singular).Under the MMSE criterion, optimal weightings are obtained starting from the following equations:

$e(n)=S 1(n)-y(n)$.

$e(n)=S I(n)-W^{H} X(n)$.

$E\left\{e(n)^{2}\right\}=E\left\{S 1(n)^{2}\right\}-W^{H} R_{x s l}{ }^{*}-W^{T} R_{x s I+} W^{T} R_{x x} W$

$\nabla_{w} E\left\{|e(n)|^{2}\right\}=-2 R_{x s l}+2 R_{x x} W=0$

$2 R_{x x} W=2 R x s_{1}$

$R_{x x} W=R_{x s_{1}}$

$W_{o p t}=R_{x x}^{-1} R x s_{1}$
It is the optimal solution of Wiener ${ }^{[5]}$ to calculate $W i$, there are several methods which we can quote two: LMS $^{[3]}$ (Least Mean Square) and SMI ${ }^{[10]}$ (Sampling Matrix Inversion).

Here user 1 is considered as a desired user and $s_{l}(n)$ is regarded as the training sequence of the desired signal.

$R_{X S I}$ represents the intercorrelation vector between the reference signal $s_{l}(n)$ and the signal vector $X(n)$. From (6), the power of the residual error under MMSE criterion of STAP system is obtained as:

$\sigma_{M M S E}^{2}(v)=1-R_{X S I}{ }^{H}(v) R_{X X}^{-1} R_{X S I}(v)$

Equation (6) represents a convolutive system ${ }^{[11]}$. Thus, we can directly apply the Fast Fourier transform FFT to equation (6). Since the autocorrelation function is an even function, therefore its Fourier transform is real and even. In the deterministic case the Fourier Transform of the autocorrelation function is the power spectral density of the original signal. The power spectrum is always positive and/or null. And it does not contain information about the phase.

$F\{w\} . F\{R x x\}=F\{R x s\}$

$F\{R x x\}=|X(f)|^{2}$

$F\{R x s\}=S(f) \cdot X(f)^{*}$

$F\{w\} . F\{R x x\}=F\{R x s\}$

$F\{R x x\}=|X(f)|^{2}$

$F\{R x s\}=S(f) \cdot X(f)^{*}$

(10)

$F\{w\}=W(f)$.

$F$ : represents Fourier transform.

(*): represents complex conjugate. Therefore the equation (10) becomes:

$|X(f)|^{2} W(f)=S(f) \cdot X(f)^{*}$

Since $|X(f)|^{2}$ is always positive and does not contain information on the phase of the signal. We can normalize its values between 0 and 1 . That will not affect the results which we can obtain.

One poses, now,

$H=|X(f)|^{2}$.

$x=W(f)$;

$y=S(f) \cdot X(f)^{*}$

Then we have the system :

$H . x=y$

Iterative techniques: Let the signal of interest be denoted as x. Often, this signal is distorted and after a transformation $\mathrm{H}$, is given by:

$y=H . x$

The problem is to estimate $\mathrm{x}$, given $\mathrm{y}$ and $\mathrm{H}$ in (13). This objective may be achieved by applying the inverse transformation $\mathrm{H}^{-1}$ to $\mathrm{y}$ to obtain $\mathrm{x}$ as follows:

$\mathrm{x}=\mathrm{H}^{-1} \mathrm{y}$

However, the solution of (14) may not be as straight forwards the equation itself ${ }^{[12,13]}$, especially when:

i. The inverse of $\mathrm{H}$ does not exist,

ii. $\mathrm{H}$ has singular points, that is $\mathrm{H}^{-1}$ has some points in its domain where it does not exist and 
iii. The problem of finding $\mathrm{H}^{-1}$ is ill-conditioned.

Under such conditions, the iterative techniques ${ }^{[13]}$ can be used. In general, iterative techniques have the followings advantages:

a. The inverse $\mathrm{H}^{-1}$ is not explicitly required and therefore the above-mentioned difficulties are circumvented.

b. Restoration can be carried out for nonlinear or shift variant degradations.

c. Nonlinear constraints can be incorporated in the restoration process.

To study the solution of $\mathrm{x}$ in (13) by iterative methods, it is assumed that $\mathrm{x}$ and $\mathrm{y}$ belong to a linear vector space $S$ which is a Banach space ${ }^{[8]}$. In such a space, the distance between any two element $\mathrm{x}$ and $\mathrm{y}$ is denoted by $d\{x, y\}$ and the norm of an element $x$ by $\|\mathrm{x}\|$. Further, in such a space Cauchy convergent sequence $\left\{x_{k}\right\}$ converges to a limit point $x$ in $S$ such that $\mathrm{d}\left\{\mathrm{x}_{\mathrm{k}}, \mathrm{x}\right\} \rightarrow 0$ as $\mathrm{k} \rightarrow \infty$.

In addition to the assumption on $\mathrm{S}$, the operator $\mathrm{T}$ on S including the distortion operator $\mathrm{H}$ in (14) has the following properties:

a. Both the domain and the range of $\mathrm{T}$ are subsets of $\mathrm{S}$.

b. An operator $\mathrm{T}$ on $\mathrm{S}$ is bounded ${ }^{[7,10]}$ if

$\mathrm{d}(\mathrm{Tx}, \mathrm{Ty}) \leq \operatorname{Md}(\mathrm{x}, \mathrm{y})$

Where $\mathrm{M}$ is a constant called the bound of $\mathrm{T}$ and is denoted by $\|\mathrm{T}\|$. If $0 \leq \mathrm{M}<\mathrm{I}$, the operator $\mathrm{T}$ is defined as a contraction operator.

c. I is the identity operator.

d. The zero operator $\Phi$ has the property $\Phi \mathrm{x}=0, \forall \mathrm{x} \in \mathrm{S}$.

e. For an iterative operator $\mathrm{T}, \mathrm{T}^{\mathrm{k}}$ would mean that the operator $\mathrm{T}$ is applied $\mathrm{k}$ times.

A generalized approach: Let the applications of an iterative technique for solving $\mathrm{x}$ in (13) generate a sequence $\left\{\mathrm{x}_{\mathrm{k}}\right\}$. An associated error sequence $\left\{\mathrm{e}_{\mathrm{k}}\right\}$ is defined as follows:

$e_{k}=x-x_{k}$

The iterative technique will be deemed to be successful if the sequences $\left\{\mathrm{x}_{\mathrm{k}}\right\}$ and $\left\{\mathrm{e}_{\mathrm{k}}\right\}$ have $\mathrm{x}$ and 0 as their respective limit points.

If, after the $\mathrm{k}^{\text {th }}$ iteration, $\mathrm{e}_{\mathrm{k}}$ is known, then the limit point $\mathrm{x}$ can be exactly calculated as:

$x=x_{k}+e_{k}$

However, in practice, $e_{\mathrm{k}}$ would not be known and at best only its estimate $\hat{e}_{\mathrm{k}}$ may somehow be computed. Using this estimate in (17), instead of the exact limit point $x$, its estimate denoted $x_{k+1}$ is obtained. Therefore, the general recursive equation for iterative techniques is derived from (17) as follow.

$x_{k+1}=x_{k}+\hat{e}_{k}$

For the purpose of evaluating the sequence of estimates $\left(\hat{e}_{\mathrm{k}}\right)$ an associated residual error sequence $\left\{\mathrm{e}_{\mathrm{y}, \mathrm{k}}\right\}$ is calculated from the observations y in (13) and iteration sequence $\left\{\mathrm{x}_{\mathrm{k}}\right\}$ as follows:

$e_{y, k}=y-H x_{k}$ if for $\mathrm{k} \rightarrow \infty, \mathrm{x}_{\mathrm{k}} \rightarrow \mathrm{x}$ then $\mathrm{Hx}_{\mathrm{k}} \rightarrow \mathrm{Hx}$ and equations (13) and (19) imply that $\mathrm{e}_{\mathrm{y}, \mathrm{k}} \rightarrow 0$ as $\mathrm{k} \rightarrow \infty$. Therefore the sequence $\left\{\mathrm{e}_{\mathrm{y}, \mathrm{k}}\right\}$ can be used as a control to test the desired convergence of $\left\{\mathrm{x}_{\mathrm{k}}\right\}$. The existing iterative techniques thus derive $\left(\hat{e}_{\mathrm{k}}\right)$ as a transformation $\mathrm{F}$ of $\left\{\mathrm{e}_{\mathrm{y}, \mathrm{k}}\right\}$ as follow:

$\hat{e}_{k}=F e_{y, k}$

The general iteration (14) can now be written as:

$x_{k+1}=x_{k}+F e_{y, k}$

Convergence: The various iterative techniques that follow (21) can be alternatively expressed in the following form of the Banach fixed point theorem ${ }^{[14]}$ :

$x_{k+1}=R x_{k}+F_{y}=T x_{k}$

Where the operators $\mathrm{R}$ and $\mathrm{T}$ are respectively given as $\mathrm{R}=(1-\mathrm{FH})$ and $\mathrm{Tx}=\mathrm{Rx}+\mathrm{Fy}$. If $T$ is a contraction operator as defined in (15), then the sequence $\left\{x_{k}\right\}$ generated by (22) converge to a unique fixed point or the limit point $\mathrm{x}$. The error at the $\mathrm{k}^{\text {th }}$ iteration or distance between the solution $x_{k}$ and the true limiting point solution $\mathrm{x}$ is:

$d\left(x_{k}, x\right)=d\left(T x_{k-1}, T x\right)$

$\leq M d\left(x_{k-1}, x\right)$

$\leq M^{k} d\left(x_{0}, x\right)$

Where the use of the definition of fixed point, that is,

$\mathrm{Tx}=\mathrm{x}$, and of equation (14) and (22) has been made. The convergence is thus linear form one step to the next iteration (23a) and follows a geometric progression with reference to the initial starting point $\mathrm{x}_{0}$ (inequality (23b)).

The proposed algorithm: For the solution of $\mathrm{x}$ in (14), equation (18) forms the basis of the iterative technique. However, in the proposed technique, $\hat{\mathrm{e}}_{\mathrm{k}}$ is not derived according to equations (19) and (20) as in the other iterative techniques. Let a transformation $\mathrm{H}_{\mathrm{k}}$ be defined as

$x_{k}=H_{k} x \quad \forall k=0 ; 1 ; \ldots$

Then, (4) can be written as

$e_{k}=\left(1-H_{k}\right) x$

$=B_{k} x$

Where

$B_{k}=I-H_{k} \forall \mathrm{k}=0 ; 1 ; \ldots$

Now, given the estimate $x_{k}$ of $x$, the estimate is obtained from (21) as:

$\hat{e}_{k}=B_{k} x_{k}$

The basic iteration equation (18) is, know, given as:

$x_{k+1}=x_{k}+B_{k} x_{k}=\left(I+B_{k}\right) x_{k}$

$=\left(2 I-H_{k}\right) x_{k}$

Where use of (26) (22) has been made in the derivation of (28b). Substituting (24) in (28b), we have:

$\mathrm{X}_{\mathrm{k}+1}=\left(2 \mathrm{I}-\mathrm{H}_{\mathrm{k}}\right) \mathrm{H}_{\mathrm{k}} \mathrm{X}$

Comparison of (24) and (29) indicates

$\mathrm{H}_{\mathrm{k}+1}=\left(2 \mathrm{I}-\mathrm{H}_{\mathrm{k}}\right) \mathrm{H}_{\mathrm{k}}$

Equations (28b) and (30) constitute the proposed technique. From (30) 
$1-H_{k+1}=1-2 H_{k}+H_{k}^{2}$

$1-H_{k+1}=\left(I-H_{k}\right)^{2}$

Equation (26) and (31) imply

$\mathrm{B}_{\mathrm{k}+1}=\mathrm{B}_{\mathrm{k}}^{2}$

Equations (28a) and (32) constitute the alternative form of the proposed technique. If the iterations are started with $\mathrm{x}_{0}=\mathrm{y}$ then (14) and (24) amply that $\mathrm{H}_{0}=$ $\mathrm{H}$. The proposed algorithm is resumed by equations (26), (28a), (32), respectively:

$B_{0}=1-H_{0}$

$x_{k+1}=x_{k}+B_{k} x_{k}$

$B_{k+1}=B_{k}^{2}$

Principle of the proposed method: The method suggested by ${ }^{[8]}$ is based on the use of the MMSE criterion which led to the form (7) and they use LMS algorithm to calculate the weighting coefficients Wi of the filters. For the validation of their results they used:

$\varepsilon_{\mathrm{STAP}}^{2}(\mathrm{l})=\frac{1}{\mathrm{~L}} \sum_{\mathrm{n}=1}^{\mathrm{L}}\left|\mathrm{S}_{1}(\mathrm{n})-\sum_{\mathrm{m}=1}^{\mathrm{M}} \mathrm{W}_{\mathrm{m}}^{\mathrm{T}}(\mathrm{l}) \mathrm{X}(\mathrm{n}-\mathrm{m}+1)\right|^{2}$

According to the equation (7), we can show that the difficulty lies especially in the inversion of the matrix Rxx. In the literature we can find several algorithms which treats the inversion of this matrix and which are especially based on the use of algorithms $\mathrm{SMI}^{[10]}$ and $\mathrm{LMS}^{[5]}$.

Moreover one improved version NLMS (Normalized LMS) was introduced by Horner ${ }^{[15]}$ but it always suffers from the problem of convergence speed $^{[7]}$.

To circumvent the problem of convergence of algorithm LMS Yang et al. ${ }^{[8]}$ have proposed the use of the sub-band STAP ${ }^{[16,17]}$. They have find that with their algorithm convergence is faster than conventional STAP. Convergence is reached in the neighborhoods of 2000 iterations $^{[8]}$.

In our work we dealt with the problem in the frequential field. In section 3 we have obtained equation (12) $H . x=y$. Witch is similar to (13).

Then we can apply the algorithm (33) and we can obtain the weights vector $W$ iteratively. The next paragraph shows the simulation results.

\section{RESULTS}

Here, the results of simulations are presented to prove the effectiveness of our algorithm in the improvement of the performances of STAP.

An ULA network of 3 omni directional identical antennas spaced of $\lambda / 2$ is used. The factor of over sampling $\mathbf{J}=2$. The scenario of several users is considered (Table 1). All the signals are binary modulated in QAM4 with raised-cosine pulse shaping filtering $\rho=0.35$. Each user presents six signals arriving by various angles of arrival to the network of antennas. The size of the signals is $\mathrm{N}=5000$ symbols and we added a noise at the reception of SNR $=100 \mathrm{~dB}$. For the convergence of our method, the steady state is reached at the end of only 10 iterations.

Table 1a: Parameters of the desired user

\begin{tabular}{llll}
\hline $\mathrm{N}^{\circ}$ & $\theta(\mathrm{deg})$ & $\tau(\mathrm{sym})$ & $\xi$ (Fading) \\
\hline 1 & -12.3 & 0 & 1.0 \\
2 & -28.0 & 0.99 & $0.02-0.84 \mathrm{i}$ \\
3 & -13.1 & 1.16 & $0.09+0.80 \mathrm{i}$ \\
4 & -0.80 & 3.89 & $-0.75-0.26 \mathrm{i}$ \\
5 & -24.0 & 5.69 & $-0.54-0.44 \mathrm{i}$ \\
6 & -26.0 & 7.41 & $-0.52-0.29 \mathrm{i}$ \\
\hline
\end{tabular}

Table 1b: Parameters of the interference user \#1

\begin{tabular}{llll}
\hline $\mathrm{N}^{\circ}$ & $\theta(\mathrm{deg})$ & $\tau(\mathrm{sym})$ & $\xi$ (Fading) \\
\hline 1 & -8.6 & 0 & 1.0 \\
2 & -12.7 & 0.65 & $0.78+0.06 \mathrm{i}$ \\
3 & -21.2 & 1.09 & $0.65-0.33 \mathrm{i}$ \\
4 & -27.2 & 6.43 & $-0.58-0.17 \mathrm{i}$ \\
5 & -10.9 & 6.69 & $0.06+0.54 \mathrm{i}$ \\
6 & -26.0 & 9.46 & $-0.39-0.34 \mathrm{i}$ \\
\hline
\end{tabular}

Table 1c: Parameters of the interference user \#2

\begin{tabular}{llll}
\hline $\mathrm{N}^{\circ}$ & $\theta(\mathrm{deg})$ & $\tau(\mathrm{sym})$ & $\xi$ (Fading) \\
\hline 1 & -6.6 & 0 & 1.0 \\
2 & -3.3 & 1.29 & $0.04+0.86 \mathrm{i}$ \\
3 & -8.7 & 1.74 & $0.26+0.76 \mathrm{i}$ \\
4 & -9.4 & 5.73 & $0.70+0.29 \mathrm{i}$ \\
5 & -14.0 & 6.47 & $0.49+0.06 \mathrm{i}$ \\
6 & -0.30 & 8.15 & $-0.37-0.25 \mathrm{i}$ \\
\hline
\end{tabular}

The Fig. 2 and 3 represents the evolution of the result according to the time of execution $\mathrm{T} 1$ for signals QAM4. We see that the recovered signal $\mathrm{S}_{1} \mathrm{r}(\mathrm{n})$ is obtained with high resolution after 10 iterations or T1 (depends on machine speed). The steady state for LMS was retched after 1000 iterations or 40 T1. Figure 4 and 5 represent the residual error power according to the number of the iterations for QAM4 signal.

Figure 3-7 represent the evolution of LMS equalization in function of the same time $\mathrm{T} 1$ that is performed by our algorithm. Thus, we can see that the LMS algorithm starts to give something after $15 \mathrm{~T} 1$. In Fig. 8 the steady state is reached by LMS algorithm after $40 \mathrm{~T} 1$.
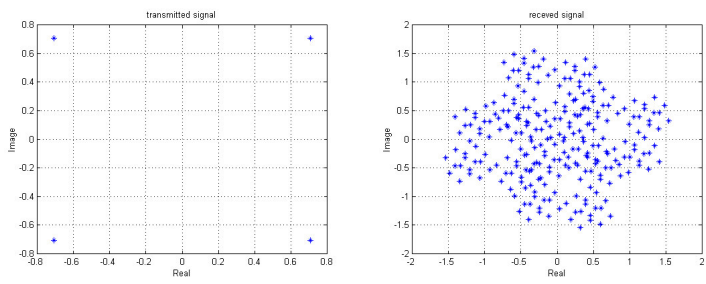

Fig. 2: Left: Transmitted signal, Right: received signal
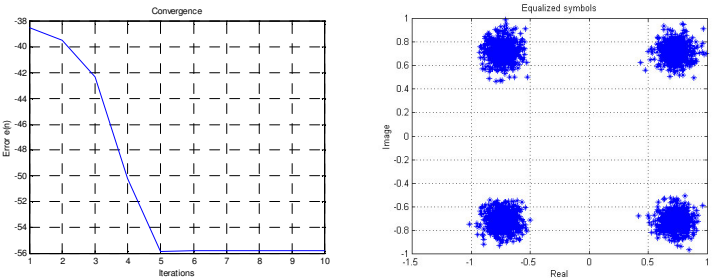

Fig. 3: Left: Residual error power for our method after time T1, Right: Equalisation result 

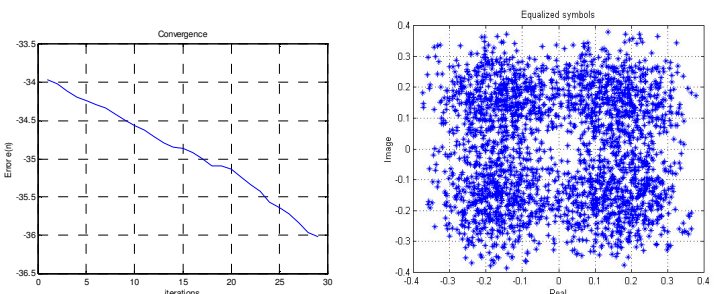

Fig. 4: Left: Residual error power for LMS after time of execution $\mathrm{T} 1$.

Right: Result of equalisation by LMS algorithm after time $\mathrm{T} 1$
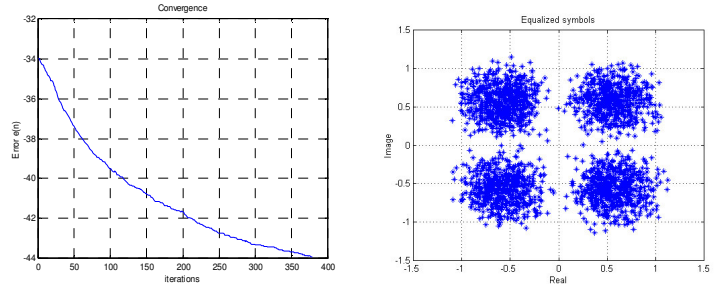

Fig. 5: Left: resudual error power. After time $t=15 T 1$, Right: Equalisation result by LMS after time $\mathrm{t}=15 \mathrm{~T} 1$
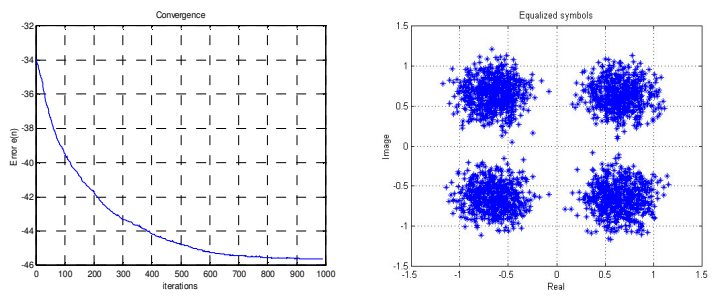

Fig. 6: After time $\mathrm{t}=35 \mathrm{~T} 1$ (LMS)
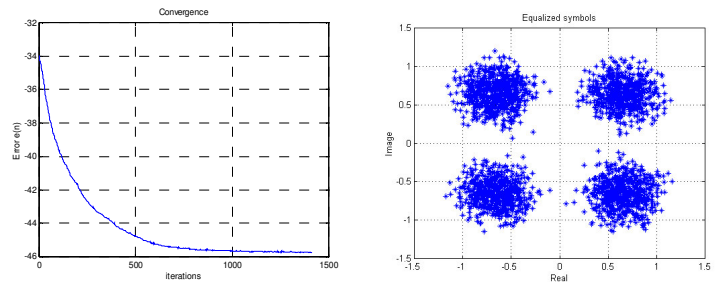

Fig. 7: After time $\mathrm{t}=50 \mathrm{~T} 1$ (LMS)

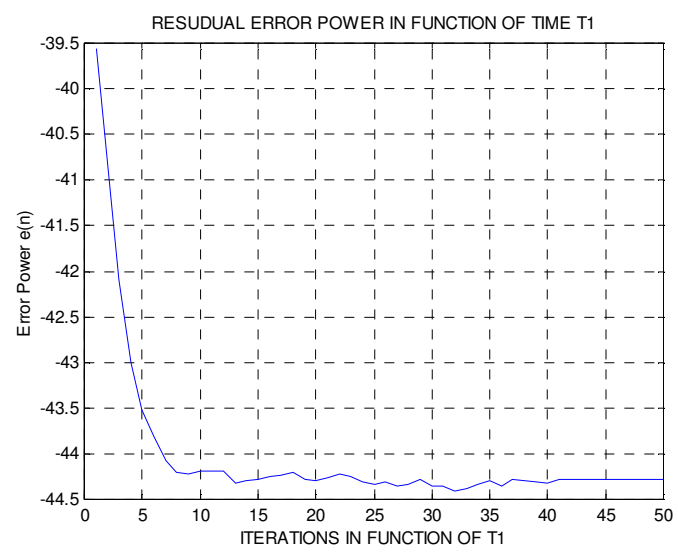

Fig. 8: The residual error power in function of time T1 for LMS

\section{CONCLUSION}

The effectiveness of our method is improved by the results so obtained. From the point of view of speed convergence our algorithm is faster than LMS, the convergence was reatched after 5 iterations,. The performance of LMS was attained after $50 \mathrm{~T} 1$, i.e., after 1500 iterations.

\section{REFERENCES}

1. Anderson, S., M. Millnert, M. Viberg and B. Wahlberg, 1991. An adaptive array for mobile communication systems. IEEE Trans. Veh. Technol., 40: 230-236.

2. Paulraj, A.J. and C.B. Papadias, 1997. Space-time processing for wireless communications. IEEE Signal Process. Mag., 14: 49-83.

3. Richard, K., 1998. Space-Time Adaptive Processing, Principles and Applications. IEE Radar, Sonar, Navigation and Avionics series, 9.

4. Ivica, S., A. Skrivervik and J.R. Mosig, 2003. Final Report: Smart Antenna Systems for Mobile Communications. Laboratoire d'Electromagnétisme et d'Acoustique. Ecole Polytechnique Fédérale de Lausanne.

5. Haykin, S., 2001. Communication Systems. 4th Edn. John Wiley \& Sons.

6. Alle-Jan van der, V., 1996. Resolution limits of blind multi-user multi-channel identification schemes-the bandlimited case. IEEE ICASSP'96, Atlanta (GA).

7. Vigneswaran, S., 2003. Spatial frequency domain NLMS algorithm for smart antenna systems. Bachelor of Engineering Thesis, Univertsity of Quensland.

8. Yang, K. et al., 2001. A signal subspace-based subband aproach to space-time adaptive processing for mobile communications. IEEE Trans. Signal Process., 49: 401412.

9. Kohno, R., 1998. Spatial and temporal communication theory using adaptive antenna array. IEEE Pers. Commun., 5: 28-35.

10. Reed, I.S., J.D. Mallett and L.E. Brennan, 1974. Rapid convergence rate in adaptive arrays. IEEE Trans. Aerosp. Electron. Syst., AES-10.

11. Robert, A.M. and T.W. Miller, 1980. Introduction to Adaptive Arrays. John Wiley \& Sons.

12. Neumaier, A., Solving ill-conditionned and singular linear systems: A Tutorial on regularization', Institut fur Mathematik, Universitat Wien, Austria.

13. Arbenz, K. and A. Wohlhauser, 1983. Analyse Numérique. Presses Polytechniques Romandes.

14. Agarwal, R.P., M. Meehan and D.O'Regan, 2001. Fixed Point theory and Applications. Cambridge University Press.

15. Homer, J., 2000. Detection guided NLMS estimation of sparsely parametrized channels. IEEE Trans. Circuit and Systems II: Analog and Digital Signal Processing, 47: 1437-1442.

16. Zhang, Y., K. Yang and M.G. Amin, Adaptive array processing for multipath fading mitigation via exploitation of filter banks," IEEE Trans.Antennas Propagat.

17. Zhang, Y., K. Yang and Y. Karasawa, 1999. Subband CMA adaptive arrays in multipath fading environment. IEICE Trans. Commun., J82-B: 97-108. 